\title{
List of figures and tables
}

\section{Figures}

6.1 From the linguistic input to the recombination of conceptual structures, with the underlying syntactic input. 233

\section{Tables}

2.1 Theories' claims and predictions. -59

3.1 Summary of the acquisition timeline -89

4.1 Populations and performance, summary 140

6.1 Theories' claims and predictions. 230 
